\title{
Induction of labour and its feto-maternal outcome
}

\author{
Mridu Sinha $^{1 *}$, Shashi Bala Arya ${ }^{1}$, Shashi Saxena ${ }^{2}$, Nitant Sood ${ }^{1}$
}

\begin{abstract}
${ }^{1}$ Department of Obstetrics and Gynecology, SRMS IMS, Bhojipura, Bareilly, Uttar Pradesh, India
\end{abstract}
${ }^{2}$ Department of PSM, SRMS IMS, Bhojipura, Bareilly, Uttar Pradesh, India

Received: 27 April 2019

Accepted: 05 June 2019

*Correspondence:

Dr. Mridu Sinha,

E-mail: sinhamridu72@gmail.com

Copyright: () the author(s), publisher and licensee Medip Academy. This is an open-access article distributed under the terms of the Creative Commons Attribution Non-Commercial License, which permits unrestricted non-commercial use, distribution, and reproduction in any medium, provided the original work is properly cited.

\begin{abstract}
Background: Induction of labour is an iatrogenic deliberate attempt to terminate the pregnancy in order to achieve vaginal delivery in cases of valid indication. It should be carefully supervised as it is a challenge to the clinician, mother and the fetus. Aim of this study was to find out common indications for IOL in a tertiary care teaching centre and its feto-maternal outcome.

Methods: An institutional based retrospective observational study was conducted to describe the prevalence of labour induction and factors associated with its outcome, during the time-period of one year from January 2018 to December 2018, at SRMS IMS, Bareilly. Logistic regression analysis was employed to assess the relative effect of determinants and statistical tests were used to see the associations.

Results: Most of the patients were primigravidas of younger age-group. Idiopathic oligohydramnios and postdatism were the commonest indications for induction of labour and Misoprost was the commonest drug used for it. Though majority had vaginal delivery, as the method was changed to combined method it was significantly associated with increased likelihood of LSCS. Similarly there was increased association with maternal cervico-vaginal tear / lacerations as the method was changed to combined type. However there were no association between post-partum hemorrhage, meconium stained liquor or fetal distress.

Conclusions: Common indications for induction of labour were oligohydramnios and postdatism. Misoprost can be safely used for induction of labour without any increased risk for LSCS or any fetal / neonatal risks.
\end{abstract}

Keywords: Fetal distress, Fetal outcome, Induction of labour, Maternal outcome, Misoprost

\section{INTRODUCTION}

Over the past several decades, induction of labour has become a common obstetric procedure, with a universal goal to ensure the best possible outcome for both mother and her newborn. Induction of labour is an iatrogenic initiation of uterine contractions in a pregnant woman after the age of fetal viability, who is not in labour to help her achieve a vaginal birth. This is done when risk of continuing the pregnancy either for the mother or for the fetus, exceeds the risk associated with induced labour and delivery. WHO recommends that induction should be performed with a clear medical indication and when expected benefits outweigh potential harms. ${ }^{1}$ The rate of induction varies by location as well as institution. According to an analytical study, it is generally less common in lower-income (4.4\%-Africa and $12.1 \%$-Asia) than higher-income countries (approx. 20\% in UK and USA), except Sri Lanka (35.5\%) and India (32\%). ${ }^{2}$ In US it has increased from $9.5 \%$ in 1990 to $22.1 \%$ in $2004 .^{3}$

Successful induction is defined as achieving vaginal delivery within 24 to 48 hours of induction of labour. Success of induction depends largely on cervical status, 
an unripe cervix has a lower likelihood of vaginal delivery.

Elective induction is induction of labour in absence of acceptable fetal or maternal indication. After 41 weeks of gestation, it is associated with a small reduction in perinatal deaths and meconium aspiration syndrome. ${ }^{4}$ However, elective induction should not be performed before 39 weeks gestation, as perinatal outcomes are less favourable. $^{5}$ Non-reassuring fetal status is a contraindication to induction of labour.

There are various methods of induction including pharmacological - most common being prostaglandins and oxytocin, and mechanical - Foley's catheter, artificial rupture of membrane etc.

Objective of this study was to know the prevalence and most common indication for induction, to find out the commonly used method for it and to evaluate the safety and effectiveness of agents and method used for it.

\section{METHODS}

This was a retrospective observational study of all pregnant ladies who were admitted for safe confinement in Obs and Gynae department of Sri Ram Murti Smarak Institute of Medical Sciences, Bareilly, during a one year span from January 2018 to December 2018. Thus, the total number of patients recruited, who satisfied inclusion-exclusion criteria, during study period were 388 .

\section{Inclusion criteria}

- Maternal age group 15-44 years

- Singleton pregnancy, reliable dates, previous regular menstrual cycles

- Cases in which gestational dating is confirmed by ultrasonography performed between 12- 22 weeks of pregnancy

- Preeclampsia

- Chorioamnionitis

- Suspected fetal compromise

- Term / preterm pre-labour rupture of membranes

- Postdates (> 41+0 weeks) or post-term (> 42+0 weeks) pregnancy

- Gestational diabetes mellitus

- Intrauterine growth restriction

- Oligohydramnios

- Gestational hypertension $\geq 38$ weeks

- Intrauterine fetal death, fetal congenital malformation.

\section{Exclusion criteria}

- Maternal age groups $<15$ and $>44$ years

- Previous LSCS or scarred uterus

- Unknown dates, irregular menstrual cycles
- All women who reported in spontaneous labour

- Cardiac diseases in pregnancy

- Placenta or vasa previa or cord presentation

- Abnormal fetal lie or presentation (e.g. transverse lie or footling breech)

- Prior classical or inverted T uterine incision

- $\quad$ Significant prior uterine surgery (e.g. full thickness myomectomy)

- Active genital herpes

- Pelvic structural deformities

- Invasive cervical carcinoma

- Previous uterine rupture.

Their obstetric records were reviewed and data was collected pertaining to their demography, obstetric history, indications for induction of labour and their maternal-fetal outcome in terms of mode of delivery, maternal and fetal complications. Logistic regression analysis was employed to assess the relative effect of determinants and statistical tests were used to see the association.

\section{RESULTS}

During the one year study period, total number of patients induced was 388, who satisfied the above-mentioned inclusion criteria. Thus with total 2803 deliveries in the institution during this period, the prevalence of induction of labour was $13.84 \%$.

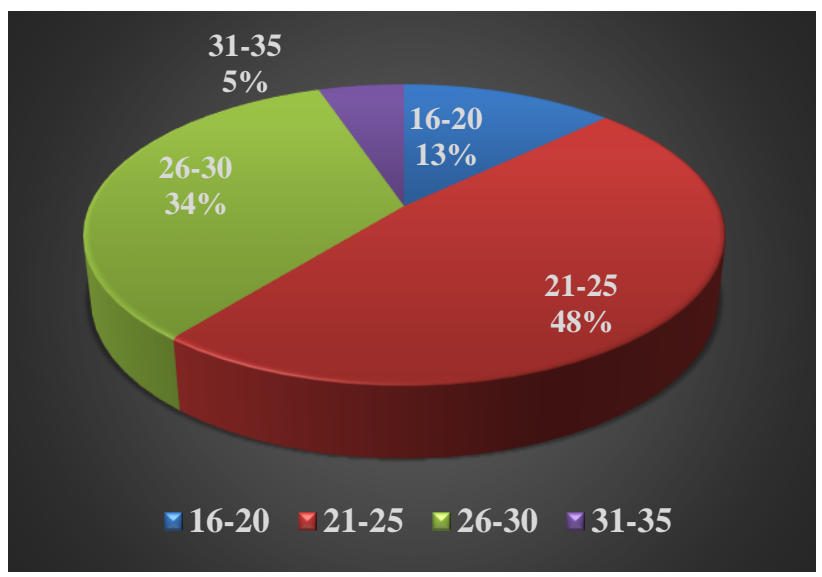

Figure 1: Age-group wise distribution of study population.

Most common age-group was 21-25years with an average age of 25.3years (Figure 1). They accounted for $48 \%$ of study population, followed by 26-30years age-group making $34 \%$, together adding up to $82 \%$. This shows that comparatively younger age-group women were induced.

According to gravidity, majority were primigravidas who constituted approx. $46 \%$ of total study population undergoing induction of labour (Figure 2). These were followed by second gravida, third gravida, fourth, fifth in that order, confirming that as the number of parities 
increases there is decreased need for induction of labour. In other words, we can say that there is more chance of going into spontaneous labour as the number of pregnancy increases.

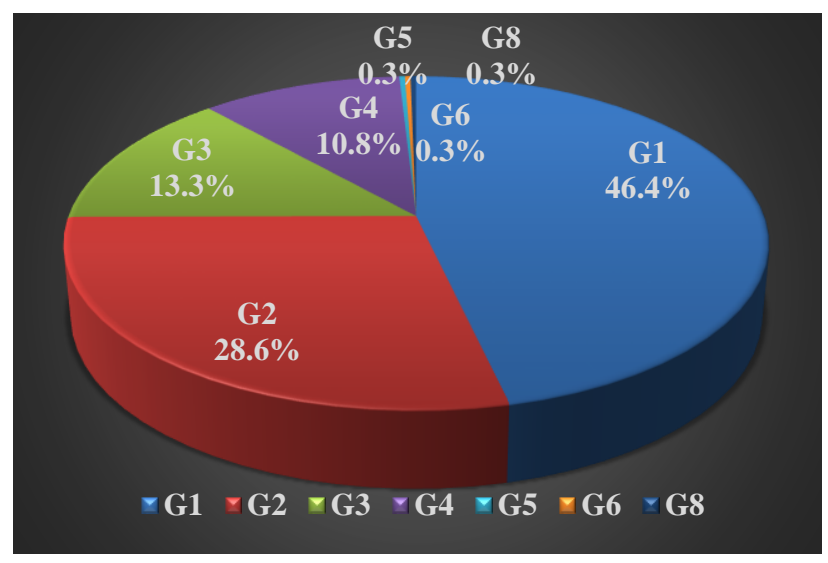

Figure 2: Gravida-wise distribution of study population.

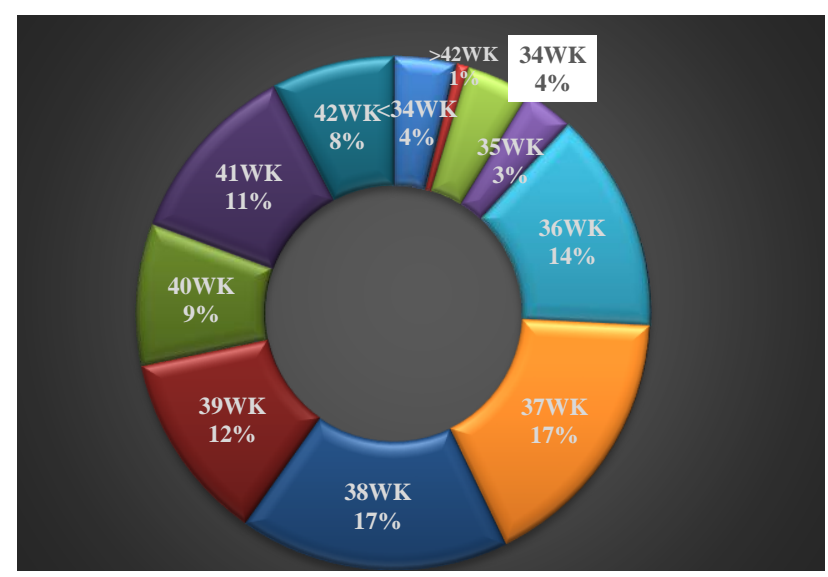

Figure 3: Distribution of study population according to period of gestation.

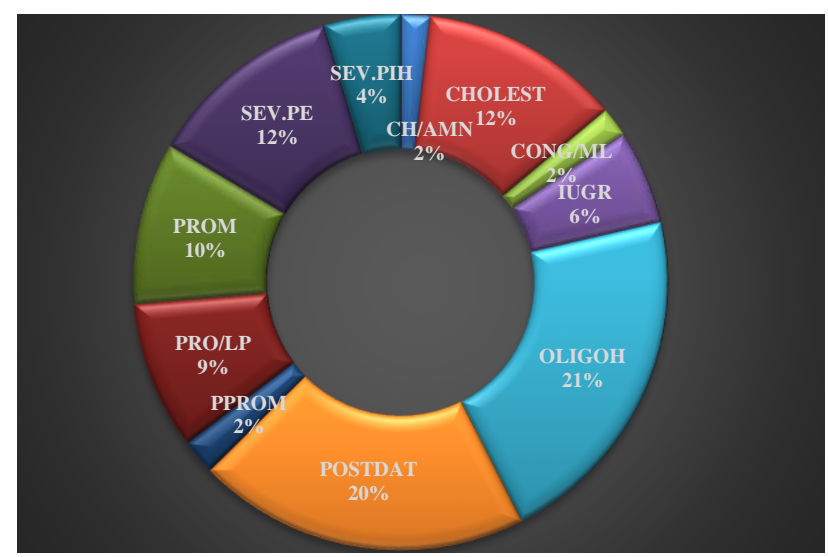

Figure 4: Distribution of study population according to indications for induction of labour.

When the patients were distributed according to timeperiod of gestation at the time of induction, the most common period of gestation was 37 and 38 weeks (34\%) followed by 39 and 36 weeks (Figure 3). This can be explained by the fact that majority waited for the fetus to reach term. Also, in untoward situations, they waited till fetal lung maturity i.e. after 34 weeks. Most of them were unbooked cases $(64 \%)$.

Regarding different reasons for which they were induced, the two most common indications were oligohydramnios $(21 \%)$ and postdatism (20\%) followed by hypertensive disorders of pregnancy $(16 \%)$ and cholestasis of pregnancy (12\%) (Figure 4). Postdated pregnancy and idiopathic oligohydramnios were the commonest reasons for induction of labour depicting that there is no role of waiting looking upon the fetal prognosis. Also, in hypertensive disorders of pregnancy, maternal prospect was considered more than the fetal.

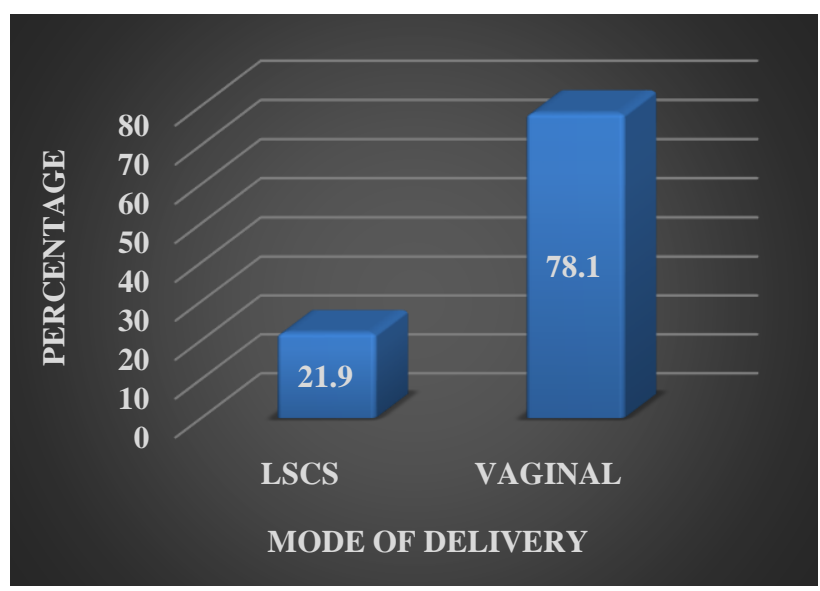

Figure 5: Maternal outcome of induction of labour.

PGE1 i.e. Misoprost alone was used in majority $(71 \%)$ of cases, PGE2 i.e. Dinoprostone gel was used in 13\% cases and combined methods i.e. PGE2 followed by PGE1 were used in $16 \%$ cases. This shows that still Misoprost is the drug of choice for induction of labour, whereas majority $(58 \%)$ had Bishop Score <6.

Pertaining to maternal outcome, $78 \%$ had vaginal delivery whereas $22 \%$ underwent LSCS (Figure 5). This finding is in concordance with studies which found that labour induction is associated with a small decreased risk of LSCS. ${ }^{6,7}$ Most common indication of LSCS was fetal distress $(46 \%)$ followed by labour disorders $(33 \%)$ and failed induction of labour (21\%), which might be governed by presence of risk factors other than the method of induction.

As per maternal complications, $8.2 \%$ had postpartum hemorrhage (PPH) and $4 \%$ had cervico-vaginal tears or lacerations but none reported rupture uterus (Figure 6). This shows that there is increase chance of both atonic as well as traumatic PPH, if a pregnant lady is artificially induced for labour. 


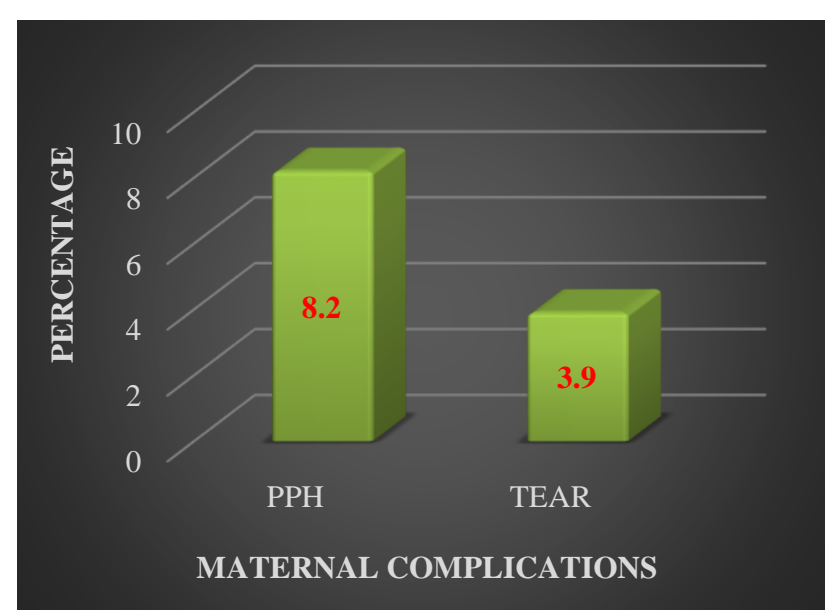

Figure 6: Maternal complications of induction of labour.

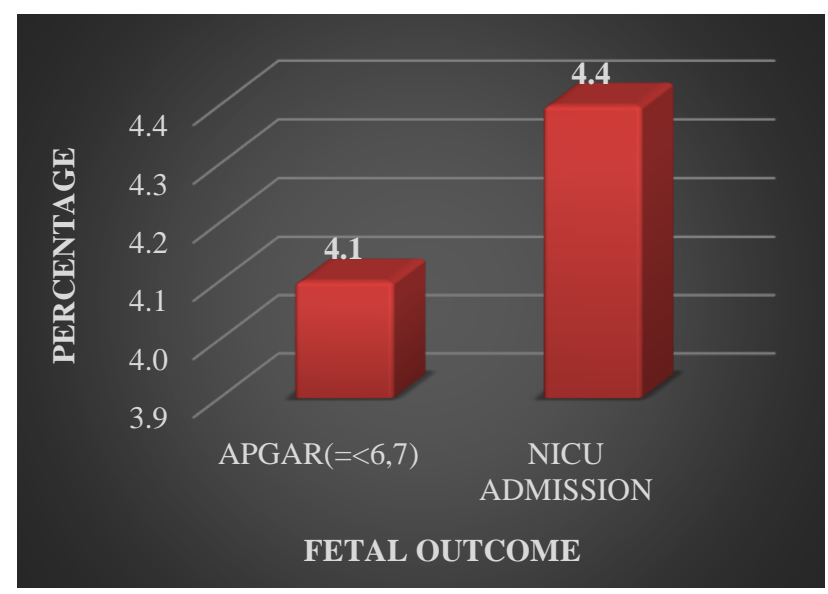

Figure 7: Fetal outcome of induction of labour.

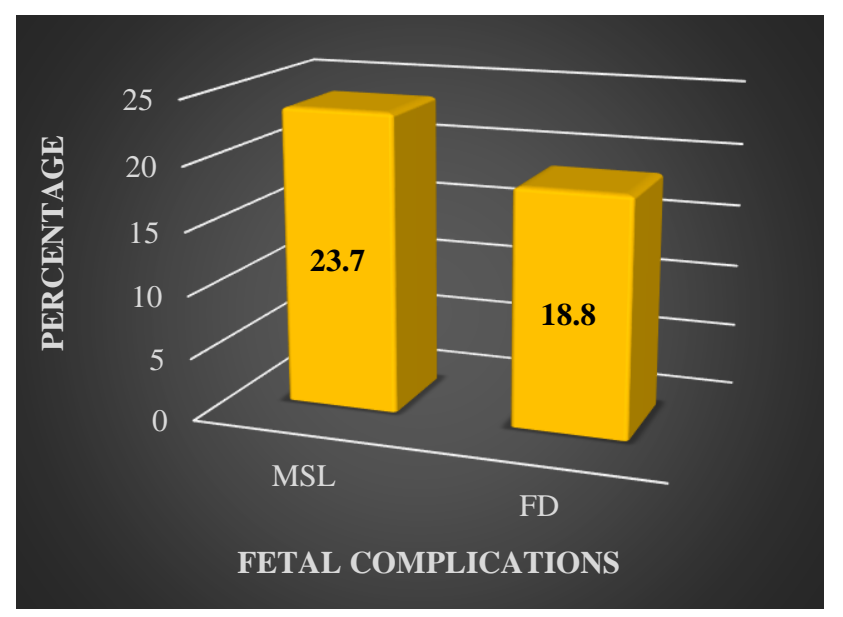

Figure 8: Fetal complications of induction of labour.

Regarding fetal outcome, only $4.1 \%$ had APGAR $<6,7$ and $4.4 \%$ needed NICU admission (Figure 7). This is almost insignificant and comparable to those with spontaneous onset of labour, justifying that labour induction has no adverse fetal effects as such. $23.7 \%$ had meconium stained liquor, but fetal distress was present in
$18.8 \%$ cases (Figure 8 ). Though there is increased chance of meconium stained liquor, it wasn't associated with fetal heart rate abnormalities in all.

Table 1: Significant association between methods of induction and mode of delivery.

\begin{tabular}{|cllll|}
\hline $\begin{array}{c}\text { Mode of } \\
\text { delivery }\end{array}$ & PGE1 & PGE2 & PGE1+E2 & $\begin{array}{l}\text { p- } \\
\text { value }\end{array}$ \\
\hline Vaginal & 223 & 38 & 42 & \\
\hline LSCS & 51 & 13 & 21 & $\mathbf{0 . 0 3 1}$ \\
\hline Total & $\mathbf{2 7 4}$ & $\mathbf{5 1}$ & $\mathbf{6 3}$ & \\
\hline
\end{tabular}

Statistically, significant association was found between methods of induction and mode of delivery. As the method of induction changes from PGE1 to PGE2 to the combined method, the likelihood of LSCS also increases from $19 \%$ to $25 \%$ and then to $33 \%$ respectively as compared to vaginal delivery $(\mathrm{p}=0.031)$ (Table 1$)$. This confirms that as we move from single method to combined methods for induction of labour, there is high chance of patient going for caesarean section, which may be related to patient inherent factors requiring multiple approaches.

Table 2: Significant association between methods of induction and cervico-vaginal tear / lacerations.

\begin{tabular}{|cllll|}
\hline Tear / & \multicolumn{2}{c|}{ Method } & \multicolumn{2}{c|}{ p- } \\
lacerations & PGE1 & PGE2 & PGE1+E2 & value \\
\hline Absent & 271 & 47 & 55 & \\
\hline Present & 3 & 4 & 8 & $\mathbf{0 . 0 0 0}$ \\
\hline Total & $\mathbf{2 7 4}$ & $\mathbf{5 1}$ & $\mathbf{6 3}$ & \\
\hline
\end{tabular}

Similarly, there was significant association between methods of induction and presence of maternal cervicovaginal tear / lacerations which is significantly highest when combined methods were used $(\mathrm{p}=0.00)$ (Table 2). Thus, chance of traumatic PPH increases if more than one method is used for induction of labour.

Table 3: No association between methods of induction and post-partum hemorrhage (PPH).

\begin{tabular}{|lllll|}
\hline \multirow{2}{*}{ PPH } & Method & & P \\
& PGE1 & PGE2 & PGE1+E2 & value \\
\hline Absent & 257 & 44 & 55 & \\
\hline Present & 17 & 7 & 8 & 0.072 \\
\hline Total & 274 & 51 & 63 & \\
\hline
\end{tabular}

But no association was found between atonic post-partum hemorrhage $(\mathrm{PPH})$ and method of induction $(\mathrm{p}=0.072)$ (Table 3). This shows that except for traumatic cause, other causes of PPH are not related to methods of induction. The cause could be inherent to patient herself or may be related to prevailing conditions or pregnancy complications. Also, there was no significant association between meconium-stained liquor (MSL) and methods of induction $(\mathrm{p}=0.892)$ (Table 4$)$. This confirms that though 
there is increased likelihood of meconium-stained liquor and fetal distress in patients undergoing induction of labour, but statistically it was not significant. Likewise, no significant association was found between fetal distress in form of fetal heart rate irregularities and methods of induction $(\mathrm{p}=0.188)$ (Table 5). This shows that cause of fetal distress may be due to pregnancyassociated conditions leading to induction of labour. Prostaglandins can be safely used for induction of labour as it has no significant adverse effect on fetal status.

Table 4: No association between methods of induction and meconium-staining of liquor (MSL).

\begin{tabular}{|l|llll|}
\hline MSL & Method & & p- \\
& PGE1 & PGE2 & PGE1+E2 & value \\
\hline Absent & 209 & 40 & 47 & \\
\hline Present & 65 & 11 & 16 & 0.892 \\
\hline Total & $\mathbf{2 7 4}$ & $\mathbf{5 1}$ & $\mathbf{6 3}$ & \\
\hline
\end{tabular}

Table 5: No association between methods of induction and fetal distress (FD).

\begin{tabular}{|lllll|}
\hline FD & Method & & & p- \\
& PGE1 & PGE2 & PGE1+E2 & value \\
\hline Absent & 226 & 43 & 46 & \\
\hline Present & 48 & 8 & 17 & 0.188 \\
\hline Total & $\mathbf{2 7 4}$ & $\mathbf{5 1}$ & $\mathbf{6 3}$ & \\
\hline
\end{tabular}

Table 6: Result of bivariate logistic regression analysis.

\begin{tabular}{|lllll|}
\hline \multicolumn{1}{c}{$\begin{array}{c}\text { Oddls } \\
\text { ratio }\end{array}$} & \multicolumn{2}{c|}{$\begin{array}{c}\text { L5\% } \\
\text { Lower }\end{array}$ C.Ifor EXP(B) } & Upper & P value \\
\hline Method & & & & 0.117 \\
\hline Method(1) & 1.624 & 0.767 & 3.440 & 0.205 \\
\hline Method(2) & 1.945 & 0.978 & 3.870 & 0.058 \\
\hline TEAR(1) & 1.621 & 0.469 & 5.601 & 0.445 \\
\hline MAS(1) & .560 & 0.187 & 1.677 & 0.300 \\
\hline FD(1) & 8.513 & 2.881 & 25.158 & 0.000 \\
\hline IUGR(1) & 1.844 & 0.896 & 3.796 & 0.097 \\
\hline
\end{tabular}

On bivariate logistic regression analysis, it was found that in case of fetal distress, the odds ratio was 8.513 which shows that odds of LSCS is 8 times more if fetal distress is present (Table 6). This justifies that irrespective of induction, chances of caesarean section increase if there is fetal distress.

\section{DISCUSSION}

According to Clinical Practice Recommendation Committee, Canada, every woman should ideally have an ultrasound, preferably in the first trimester, to confirm gestational age. (I-A) Women should be offered induction of labour between $41+0$ and $42+0$ weeks as this intervention may reduce perinatal mortality and meconium aspiration syndrome without increasing the
Caesarean section rate. (I-A) Women who chose to delay induction > 41+0 weeks should undergo twice-weekly assessment for fetal well-being. (I-A). ${ }^{8}$ Though in this study, induction of labour was done due to certain maternal and/or fetal risk factors, many studies including Cochrane database of systematic reviews support for elective induction of labour at term in low risk women and concluded that a policy of labour induction at or beyond term compared with expectant management is associated with fewer caesarean sections and fewer perinatal deaths..$^{9-11}$ Other studies say that induction of labour at 39weeks in low risk nulliparous women did not result in a significantly lower frequency of composite perinatal outcome. ${ }^{12,13}$ In obese nulliparous women elective induction after 39 weeks is associated with reduced maternal and neonatal morbidity. ${ }^{14}$

In gestational diabetes mellitus, routine induction at 39 weeks is associated with low risk of caesarean delivery compared with expectant management but may increase risk of neonatal ICU admission if done before 39 weeks. ${ }^{15,16}$ In Intrahepatic cholestasis of pregnancy early induction of labour at 37 weeks seem to be justified and favored by an expected major reduction in ICP stillbirth risk. ${ }^{17}$

Prostaglandins are commonly preferred agent for induction of labour and difference in mechanism of action between Misoprost (PGE1) and Dinoprostone (PGE2) should be considered to optimize outcome. ${ }^{18}$ Low dose vaginal Misoprost appears to be more effective as it requires less oxytocin augmentation, however Dinoprostone has lower incidence of hyperstimulation and tachysystole. ${ }^{19-21}$ Recently, a study has shown that Misoprost vaginal insert achieves more vaginal delivery within 24hours as compared to Misoprost vaginal tab but at the cost of tachysystole. ${ }^{22}$

Limitation of this study is that since it's a retrospective observational study, more prospective studies are required and it has not included cases electively induced, i.e. without any maternal or fetal indication.

\section{CONCLUSION}

Induction of labour should be favoured to expectant management wherever it's medically indicated as well as non-medically indicated at term as it does not increase the risk of Caesareans section nor any fetal or neonatal effects. Prostaglandins are the drug of choice, in which Misoprost achieves more vaginal delivery as compared to Dinoprostone but it should be done under careful supervision.

\section{ACKNOWLEDGMENTS}

Authors would like to thank the record-keeping department of SRMS IMS, for their continuous unconditional support. 
Funding: No funding sources

Conflict of interest: None declared

Ethical approval: Not required

\section{REFERENCES}

1. WHO recommendations for induction of labour [PDF] who.int. World Health Organization - 2011 apps.who.int. Available at: http://whqlibdoc.who.int/publications/2011/9789241 501156_eng.pdf.

2. Vogel JP, Souza JP, Gulmezoglu AM. Patterns and outcomes of induction of labour in Africa and Asia: a secondary analysis of the WHO Global survey on maternal and neonatal health. PLoS One. 2013;8(6):e65612.

3. Laughon SK, Zhang J, Grewal J, Sundaram R, BeaverJ, Reddy UM, et al. Induction of labour in a contemporary obstetric cohort. Am J Obstet Gynecol, 2012;206:486.e1-9.

4. Gulmezoglu A, Crowther C, Middleton P, Heatly E. Induction of labour for improving birth outcomes for women at or beyond term. Cochrane Database Syst Rev. 2012;(6):CD004945.

5. Stock S, Ferguson E, Duffy A, Ford I, Chalmers J, Norman JE. Outcomes of elective induction of labour compared with expected management: populationbased study. BMJ. 2012;344:e2838.

6. Little SE, Caughey AB. Induction of labor and cesarean: What is the true relationship? Cli Obstet Gynecol. 2015;58(2):269-81.

7. Tolcher MC, Holbert MR, Weaver AL, McGree ME. Predicting cesarean delivery after induction of labor among nulliparous women at term. Obstet Gynecol. 2015;126(5):1059-68.

8. Leduc D, Biringer A, Lee L, Jessica Dy. Induction of Labour: Review. SOGC Clinical Practice Guidelines. J Obstet Gynecol. 2015;37(4):380-1.

9. Middleton P, Shepherd E, Flenady V, McBain RD, Crowther CA. Planned early birth versus expectant management (waiting) for prelabour rupture of membranes at term (37 weeks or more). Cochrane database of systematic reviews. 2017(1).

10. Saccone G, Berghella V. Induction of labor at full term in uncomplicated singleton gestations: a systematic review and metaanalysis of randomized controlled trials. Am J Obstet Gynecol. 2015;213(5):629-36.

11. Bailit JL, Grobman W, Zhao Y, Wapner RJ, Reddy UM, Varner MW et al. Nonmedically indicated induction vs expectant treatment in term nulliparous women. Am J Obstet Gynecol. 2015;212(1):103.e17.

12. Grobman WA, Rice MM, Reddy UM, Tita ATN, Silver RM, Mallet G, et al. Labor induction versus expectant management in low-risk nulliparous women. N Engl J Med. 2018;379(6):513-23.

13. Sinkey RG, Lacevic J, Reliic T, Hozo I, Gibson KS, Odibo AO, et al. Elective induction of labor at 39 weeks among nulliparous women: The impact on maternal and neonatal risk. PLoS One. 2018;13(4):e0193169.

14. Gibbs Pickens CM, Kramer MR, Howards PP, Badell ML, Caughey AB, Hoque CJ. Term elective induction of labor and pregnancy outcomes among obese women and their offspring. Obstet Gynecol. 2018;131(1):12-22.

15. Vilchez GA, Dai J, Hoyos LR, Gill N, Bahado-Singh R, Sokol RJ. Labor and neonatal outcomes after term induction of labor in gestational diabetes. J Perinatol. 2015;35(11):924-9.

16. Melamed N, Ray JG, Geary M, Bedard D, Yang C, Spraque A, et al. Induction of labor before 40 weeks is associated with lower rate of cesarean delivery in women with gestational diabetes mellitus. Am J Obstet Gynecol. 2016;214(3):364.e1-8.

17. Friberg AK, Zingmark V, Lyndrup J. Early induction of labor in high-risk intrahepatic cholestasis of pregnancy: what are the costs? Arch Gynecol Obstet. 2016;294(4):709-14.

18. Bakker R, Pierce S, Myers D. The role of prostaglandins $\mathrm{E} 1$ and $\mathrm{E} 2$, dinoprostone, and misoprostol in cervical ripening and the induction of labor: a mechanistic approach. Arch Gynecol Obstet. 2017;296(2):167-79.

19. De Bonrostro Torralba C, Teiero Cabreias EL, Envid Lazaro BM, Franco Royo MJ, Roca Arquillue M, Campillos Maza JM. Low-dose vaginal misoprostol versus vaginal dinoprostone insert for induction of labor beyond 41st week: a randomized trial. Acta Obstet Gynecol Scand. 2019:PMID:30723912.

20. Liu A, Lv J, Hu Y, Lang J, Ma L, Chen W. Efficacy snd safety of intravaginal misoprostol versus intracervical dinoprostone for labor induction at term: a systematic review and meta-analysis. J Obstet Gynaecol Res. 2014;40(4):897-906.

21. Wang L, Zheng J, Wang W, Fu J, Hou L. Efficacy and safety of misoprostol compared with dinoprostone for labor induction at term: a metaanalysis. J Matern Fetal Neonatal Med. 2016;29(8):1297-307.

22. Daniele B, Saskia VW, Anda-Petronela R, Maria LG, Luigi R, Martin M, et al. Misoprostol vaginal insert versus misoprostol vaginal tablets for the induction of labour: a cohort study. BMC Pregnancy Childbirth. 2018;18:149.

Cite this article as: Sinha M, Arya SB, Saxena S, Sood N. Induction of labour and its feto-maternal outcome. Int J Reprod Contracept Obstet Gynecol 2019;8:2748-53. 